\title{
Défis D’une Orthodoxie Éthique Et Académique Chez Les Universitaires Congolais (RDC)
}

\author{
Salomon Mampeta Wabasa \\ Professeur au Département de Sociologie, Université de Kisangani, RDC. \\ Fraternel Amuri Misako \\ Professeur au Département de Sciences politiques et administratives, \\ Université de Kisangani, RDC.
}

doi: 10.19044/esj.2017.v13n14p59 URL:http://dx.doi.org/10.19044/esj.2017.v13n14p59

\begin{abstract}
In the Democratic Republic of the Congo (DRC), the huge gap clearly observed today, but which in fact is the result of a cumulative process of recent decades between what is expected and what is done on academic freedom, is a cause for concern for African learned community and beyond. Trying to impose orthodoxy as reference, both ethically and academically in the Congolese changing university space, presupposes to consider the governance (nature/quality) of Congolese society in which the university is only seen as one of the main observation windows. As a prerequisite for successful reimplementation of the professional codes of ethics among scholars, we believe that any awareness campaign would not cause the breakup of disreputable practices dominating the Congo's higher education if courageous, even unpopular but salutary reforms are not undertaken upstream. Even if scholars are to be questioned on their duties (Social Responsibility), it remains that their material conditions of living and working are not conducive to the rigorous application of ethical and professional principles for an effective exercise of academic freedom as a right. Material misery would induce moral misery and intellectual poverty, thus trapping academic freedom.
\end{abstract}

Keywords: Academic Freedom, Challenge, Ethical and Academic Orthodoxy, Governance, Scholar

\section{Résumé}

En République démocratique du Congo (RDC), l'écart visiblement énorme, observé aujourd'hui, mais qui en fait n'est que le résultat d'un processus cumulatif des dernières décennies entre ce qui est prévu et ce qui se fait en matière de liberté académique, constitue un motif d'inquiétude 
pour la communauté savante africaine, voire au-delà. Prétendre imposer l'orthodoxie comme référence, à la fois sur le plan éthique et sur le plan académique dans l'espace universitaire congolais en mutation, présuppose de s’interroger sur la gouvernance (nature/qualité) de la société congolaise dont l'université ne représente qu'une des principales fenêtres d'observation. En tant que préalable au succès de ré-implémentation des codes d'éthique professionnelle parmi les enseignants-chercheurs, nous pensons qu'aucune campagne de sensibilisation ne saurait provoquer la rupture des pratiques peu recommandables dominant le secteur de l'enseignement supérieur congolais si des réformes courageuses, voire impopulaires mais salutaires, ne sont pas entreprises en amont. Même si les enseignants-chercheurs doivent être interpellés sur leurs devoirs (responsabilité sociale), il reste que leurs conditions matérielles de vie et de travail ne sont pas de nature à favoriser l'application rigoureuse des principes éthiques et professionnels pour un exercice effectif de la liberté académique en tant que droit. La misère matérielle induirait la misère morale et la misère intellectuelle, piégeant ainsi la liberté académique.

Mots-clés : Défi-gouvernance, liberté académique, orthodoxie éthique et académique, universitaire

\section{Introduction}

«Les hommes n'acceptent le changement que dans la nécessité et ils ne voient la nécessité que dans la crise. » (Jean Monnet)

L’université et les universitaires en République Démocratique du Congo (RDC) sont, plus que jamais, confrontés à beaucoup de problèmes majeurs de leur existence. Evoquant la question de l'identité nationale de l'Université congolaise dans l'espace mondial de la connaissance, Jacqueline Bergeron (2012) constate que les problèmes complexes qui se posent aujourd'hui avec acuité à l'enseignement supérieur congolais nécessitent l'implication d'une volonté politique. Telle constatation fait transparaître en filigrane, une inquiétude par rapport aux enjeux et défis de la gouvernance en général dans le pays visé. Du côté des officiels congolais, Théophile Mbemba Fundu di Luyindu, alors ministre de l'Enseignement Supérieur et Universitaire, s'est interrogé sur les chances de succès de la réforme en cours dans son secteur, à l'occasion de l'ouverture de l'année académique 20152016. Celui-ci n’a pas caché son embarras face à l'ampleur des problèmes à la fois académiques et éthiques, comme cela transparaît dans ce cri interpellateur : 
« Mais comment y parvenir si déjà nos établissements ne respectent pas le calendrier académique et continuent à inscrire les étudiants à la veille des sessions et si nous n'appliquons pas la rigueur qu'impose la formation universitaire au moment des délibérations ? Comment pourrions-nous asseoir une bonne réputation académique et scientifique, nous permettant de réapparaître sur le classement des universités africaines si certains enseignants continuent à imposer «le droit d'auteur » et d'autres pratiques avilissantes comme conditions pour que l'étudiant réussisse à l'évaluation d'une matière enseignée ? Même si la politique d'essaimage a permis l'ouverture de nombreux établissements de l'ESU à travers le pays, comment rester indifférents aux très mauvaises conditions dans lesquelles les enseignements sont dispensés, surtout à l'intérieur du pays ? » (Forum des As, 2015).

Quelle que soit leur ampleur, il convient de noter que les problèmes se résument par le phénomène bien connu des sociologues, appelé « anomie ». Cette dernière est appréhendée en tant que situation où les normes sociales existent mais, sans avoir été abrogées puisque toujours reconnues, ne sont plus ou pas respectées par les membres d'une communauté donnée qui les a adoptées ou consacrées. Ici, c’est la communauté universitaire ou, restrictivement, la communauté savante. Ce phénomène, qui avait attiré l'attention d'Emile Durkheim (1897, 1967), mérite un regard critique dans le contexte congolais, surtout lorsqu'il s’agit de réfléchir sur les conditions de perte et les possibilités de reconquête par l'institution universitaire, de ses lettres de noblesse. Celles-ci, pour les caractériser d'une façon arbitraire car étroite, se traduisent notamment par la liberté académique attachée à la personne des enseignants-chercheurs, les universitaires, qui perpétuent sa tradition et son existence depuis la nuit des temps $^{20}$.

Par sa mission d'organiser l'enseignement et la recherche scientifique en fonction de besoins de la société, toute université peut légitimement revendiquer son statut universel. Cependant, dans les faits, il n'est pas évident que cette vérité conventionnelle se vérifie dans tous les cas. Telle constatation nous inspire d'engager la discussion sur la situation particulière, sinon atypique, des universités et des universitaires en RDC, insistant sur la question cruciale de l'opportunité de parler de l'orthodoxie éthique et académique dans le contexte critique dudit pays.

Les enseignants-chercheurs congolais, dans leur majorité, ressentent un malaise certain de lier orthodoxie éthique et orthodoxie académique, deux

\footnotetext{
${ }^{20}$ Les enseignants-chercheurs ne sont pas les seuls à exercer la liberté académique. Il s’agit cependant d'un choix délibéré pour parler essentiellement de la catégorie « enseignantschercheurs ».
} 
exigences qui sont liées comme les deux faces d'une même médaille. Pour en démontrer les aspects essentiels et spécifiques à base de données empiriques, il nous faut dans un premier temps circonscrire le constat: dresser un bref procès-verbal de carence d'actions quand bien même le contexte est favorable en termes de faits et événements suscitant l'intervention des universitaires. Dans un deuxième temps, le papier aborde dans leurs grandes lignes les défis en termes de contraintes à la mise en œuvre d'une logique orthodoxe, donc une application stricte des principes éthiques et professionnels (académiques) par les universitaires en RDC. Enfin, il sera question de conclure la réflexion par quelques brèves propositions en guise de thérapeutique en faveur de l'effectivité dans l'exercice de la liberté académique en contexte congolais.

\section{Cadre méthodologique}

Il est une évidence forte que faire la critique de sa propre organisation d'appartenance, est en lui-même un premier défi. Beaucoup d'autres chercheurs l'attestent sans réserve. En effet,

« Etre universitaire, et analyser la situation des universitaires, peut apparaître, au prime abord, incompatible avec la distance nécessaire à ce travail. Le premier à s'y risquer, en France, Pierre Bourdieu, insistait sur le fait qu'il avait longuement hésité avant de faire paraître son livre Homo Academicus(...). Il est pourtant possible d'appréhender cette profession, comme n'importe quelle profession, en utilisant les outils généralement admis dans les recherches en sciences sociales et humaines ». (Marie-Françoise et Fave-Bonnet, 2002, p. 31).

Forts de cette constatation pertinente, nous avons été à même de poursuivre l'analyse des défis soulevés par l'applicabilité de l'orthodoxie à l'univers académique congolais. En particulier, nous nous sommes basés sur les entretiens libres avec des acteurs intéressés du secteur de l'enseignement supérieur et universitaire pour soutenir notre argumentation à travers ce papier. Nous avons ainsi échangé, au fil des ans, avec les enseignants de différentes institutions, mais également, nous nous sommes entretenus avec les gestionnaires desdites institutions. A la technique d'entretien, nous avons joint l'observation participante, étant donné que nous faisons nous-mêmes partie du corps enseignant congolais, même si nous sommes affiliés à l’Université de Kisangani, champ empirique qui nous a inspiré les premières observations pour la réalisation de la présente étude. C'est grâce à cette technique que nous avons pu appréhender les réalités éthiques et académiques dans les institutions de l'enseignement supérieur et universitaire de la RD Congo. 
Enfin, la technique documentaire a été mobilisée pendant la quête de publications relatives à l'orthodoxie éthique et académique en Afrique, en général, et en RDC, en particulier. Des lectures croisées sur un certain nombre d'expériences, nous avons acquis le réflexe de penser les problèmes en termes comparatifs, ce qui nous a permis de saisir la complexité particulière de ceux-ci.

Dans notre entendement, plutôt que de parler longuement de définitions de la liberté académique ${ }^{21}$ en lui consacrant une section spécifique, qu'il suffise de rappeler que bien des écrits existent sur le sujet, qu'il s'agisse du niveau mondial, africain ou congolais ${ }^{22}$. Voici le résumé de l'excellente contribution d'André Mbata Betukumesu Mangu, donnant un aperçu juridico-historique et proposant un cadre conceptuel dynamique des libertés académiques en Afrique et en RDC à travers une analyse des situations réelles:

«Après avoir cité les instruments fondamentaux sur les libertés académiques et intellectuelles que sont la Déclaration de Lima, la Déclaration de Dar-es-Salaam, la Déclaration de Kampala, l'article conceptualise lesdites libertés dans le cadre plus large des droits de l'homme et de la démocratie, puis passe en revue l'implantation de l'université en RDC (1954-1971), son étatisation et sa récupération par le parti unique (1971-1981), l'action du libéralisme autoritaire et du programme d'ajustement structurel sur elle (1981-1990), le rendezvous manqué des libertés académiques (1990-2004). Quant aux artisans des violations des libertés académiques en RDC, ils ont pour noms l'État, les églises, les propriétaires des établissements d'enseignement, la communauté universitaire. Certes, les libertés académiques sont des droits, mais elles comportent aussi des devoirs. » (Mbata, 2005, p. 35)

\section{De la responsabilité sociale des universitaires congolais : quand la carence d'actions trahit le contexte et le concept de liberté académique}

De prime abord, il sied de noter que notre analyse s'oriente vers la démystification des pratiques universitaires contredisant l'orthodoxie sur le plan éthique. Autrement dit, nous constatons que les prescriptions ou les règles techniques et morales dans les universités congolaises ne sont plus rigoureusement respectées et ce, pour des raisons évidentes. En effet, la plupart des enseignants-chercheurs travaillent pour leurs intérêts personnels,

${ }^{21}$ Nous en parlons au singulier dans ce papier pour en conserver le sens anglo-saxon (Academic Freedom) d'unicité malgré la diversité des libertés, comme le consacrent les francophones.

${ }^{22}$ Particulièrement, pour la RDC, nous renvoyons les lecteurs à lire avec intérêt une synthèse systématique et éclairante réalisée par André Mbata Betukumesu Mangu à ce sujet (2005). 
au-delà de ceux qualifiés d'ordre éthique qui, en principe, garantissent le prestige de la science. Le domaine éthique constitue, de ce fait, le premier axe de notre démarche à ce niveau.

Le second axe nous conduit à l'examen des défis liés à l'activité professionnelle, le métier d'enseignant-chercheur, sans forcément tourner le regard vers l'éthique, bien que les deux axes soient intimement liés. L'orthodoxie académique est donc celle qui s'applique directement et pleinement aux professeurs (es) qui ont pour tâche l'enseignement, la recherche, la création, ainsi que la participation aux différentes instances universitaires et les services à la société. Elle leur permet d'accomplir ces tâches sans être assujettis à des pressions ou censures et ce, quels que soient l'orientation de leur pensée, leur style de vie, leur origine ethnique, leur langue, leur sexe, leur orientation sexuelle, leur handicap physique, leurs opinions et actions politiques ou religieuses, leur âge, leur état civil (Landry, 2001). En fait, la reconnaissance d'une vaste liberté académique implique-telle que l'université se consacre exclusivement à la recherche pure, c'est-àdire à une recherche de la Vérité au sein de laquelle n’interviendrait aucune valeur « non scientifique » (politique, sociale, religieuse, culturelle...). Dans la mesure où, comme le pensait Hannah Arendt, «la pensée spéculative dérange rarement qui que ce soit » (Arendt, 1991, p. 65) ${ }^{23}$, la question de la liberté académique semble concerner la liberté des universitaires à prendre position et non simplement leur liberté d'enseigner et de rechercher « comme bon leur semble ».

L'université paraît devoir se définir par sa liberté d'autant plus qu'elle se situe dans la vie publique et ne s'abrite pas derrière une science pure. Quitte à ce que l'université adopte une définition plus polémique, voire tout à fait politique, de la liberté académique. Ainsi, en lui reconnaissant une liberté de critique, l'Etat démocratique offrirait à l'université une tribune particulière, comme s’il reconnaissait la valeur et la possibilité de la dissidence, se garantissant à lui-même la condition de sa légitimité en tant qu'Etat démocratique. Et pourtant, il n'est pas acceptable et il n'est vraisemblablement pas indiqué que les universités se contentent de "répondre" aux sollicitations diverses de la société comme cela a été dit pendant la Conférence mondiale sur l'enseignement supérieur à Paris (Unesco, 1998). Dans quelle mesure alors les universités congolaises

\footnotetext{
${ }^{23}$ Arendt commente les propos de Kant, issus de la Critique de la raison pure: «Les gouvernements, s’ils jugent bon de se mêler des affaires de l'université, feraient mieux de « favoriser la liberté d'une telle critique [...] que de soutenir le ridicule despotisme des écoles qui jettent les hauts cris sur un danger public quand on déchire leur toile d'araignée dont le public n'a jamais eu connaissance et dont, par conséquent, il ne peut sentir la perte » (Kant, Critique de la raison pure, Paris, Gallimard, coll. de la Pléiade, p. 751).
} 
peuvent-elles prétendre à l'orthodoxie éthique et académique dans ce contexte?

Pour Allard J. et Puig de la Bellacasa M. (2005) la notion de liberté académique est envisagée comme une responsabilité et un engagement des individus ou des groupes par rapport à une institution située dans une société déterminée. Ainsi, dans l'ensemble, la liberté académique n'est pas comprise ou défendue comme un privilège mais comme une liberté responsable. Ensuite et par conséquent, le renversement éventuel d'une liberté académique / privilège ne doit pas entraîner la soumission de l'université au « nouvel ordre mondial » et aux pouvoirs afférents - économiques pour la plupart.

La question essentielle que les universitaires congolais doivent se poser aujourd'hui, est celle de savoir comment assumer les responsabilités dévolues aux universités par la société, tout en préservant la tradition notoire d'indépendance et de service objectif. Les institutions d'enseignement supérieur et universitaire sont actuellement et globalement traversées par plusieurs défis liés aux différents changements survenus, suite à la force même du changement et à la mondialisation.

En RDC, se consolide l'essaimage des institutions d'enseignement supérieur et universitaire à travers les provinces. Logiquement, cette politique d'essaimage (Otemikongo et Losumbe, 2001) devrait être accompagnée par une diffusion proportionnelle des connaissances, du savoir scientifique. Paradoxalement, plus il y a naissance d'universités, plus la société congolaise connaît des problèmes qui demeurent pendants car jamais résolus de manière effective, sinon définitive. Pourtant, les universitaires ont la responsabilité d'éclairer la société. C'est étonnant que dans ce contexte riche en faits et événements critiques, les chercheurs s'empêchent d'écrire, de diffuser le savoir. Plusieurs événements nécessitant l'intervention des universitaires ont surgi et continuent à surgir en RDC. Cependant, très peu ont été documentés parce que la plupart des universitaires se sont réservés de jouer leur rôle de premier plan. Ils n'ont pas pris en main leurs responsabilités, notamment celle d'éclairer l'opinion sur les faits aussi importants que les différentes rébellions, le changement malvenu de la Constitution, les tentatives de retoucher la loi électorale, l'organisation des concertations nationales et, tout récemment, les péripéties d'un dialogue national commencé mais qui n'en finit pas.

Ces différents événements survenus, ou potentiels, devraient conférer aussi à la production et à la diffusion des connaissances une fonction clé, celle de déterminer la place et l'insertion de la RD Congo dans le procès international de connaissances. Malgré les limites des efforts, les rares productions de chercheurs ont tout de même permis à certaines universités 
congolaises d'occuper des places modestes dans le classement africain ${ }^{24}$ (Journals Consortium, 2015).

Chose curieuse, toutefois, c'est que ces faits sont le plus souvent mieux documentés par des chercheurs étrangers. Du coup, la communauté universitaire voit ses responsabilités s'accroître considérablement, alors que la production demeure quasi-inexistante. Il y a, là, un problème de liberté académique des universitaires, mais également celui de l’autonomie des universités. Nombre d'événements, susceptibles de faire la préoccupation des universitaires, se sont révélés des sujets tabous, tout simplement parce que les franchises universitaires sont violées par les autorités politiques.

Les universitaires ne se sentent pas protégés et sécurisés car les espaces intellectuels et physiques qui leur sont destinés, en raison de franchises universitaires, sont souvent violés. Un phénomène anodin mais significatif renseignant sur la problématique des franchises universitaires en RDC, s’observe à travers la réaction épidermique et quasi-synchronisée de rejet chez les étudiants face à la présence inhabituelle des éléments de forces de l'ordre sur le campus. Dans nombre de circonstances, notamment en période trouble, les faits de violence sont toujours à craindre. Par exemple, lorsque des policiers sont déployés aux entrées principales du campus, ou simplement l'encerclent, pour empêcher les étudiants de descendre dans les rues en guise de protestation contre une mesure de l'autorité urbaine ou du gouvernement central, l'affrontement entre les deux parties est toujours à craindre. Le cas emblématique de ces trois dernières décennies, demeure ce qui était connu sous le nom de « Massacre de Lubumbashi ${ }^{25}$ ».

Parmi les universitaires qui ont écrit ces dernières années, il y en a qui se sont livrés à encenser certaines personnalités politiques, afin de gagner leur sympathie et qui, en retour, bénéficient des postes politiques et d'autres avantages y afférents. Il est dès lors important de se poser la question de savoir dans quelle mesure les universitaires congolais (professeures et professeurs) ont-ils conscience de la menace qui pèse sur l'autonomie des institutions au sein desquelles ils œuvrent et de l'importance de cette autonomie pour l'ensemble de la société? Jusqu’à quel point perçoivent-ils le carcan de plus en plus étroit dans lequel on veut insérer leur travail d'enseignement et de recherche, brimant ainsi leur liberté académique ? Et

\footnotetext{
${ }^{24} \mathrm{Il}$ est toutefois déconcertant que la première grande université publique congolaise, Université de Kinshasa (UNIKIN), occupe une place loin derrière la première centaine des meilleures en Afrique : $153^{\mathrm{e}}$ rang alors que University of Zimbabwe, du Zimbabwe, l'un des derniers pays décolonisés d'Afrique (indépendant en 1980), se classe $35^{\mathrm{e}}$.

${ }^{25}$ Il semble, de l'avis de nombreux témoins et observateurs avertis, que ce qui fut appelé « Massacre des étudiants de l’Université de Lubumbashi au Katanga dans les années 90 », n’en était pas un ; ce fut plutôt un montage bien grossier pour disqualifier le régime Mobutu, pratique qui avait déjà réussi contre le gouvernement Lumumba, accusé d’avoir commandité des massacres au Bakwanga sous la Première République.
} 
pourtant, la liberté universitaire reconnaît aux professeures et professeurs le droit de décider de leurs opinions et de l'expression de celles-ci, de choisir l'objet de leur recherche et leurs méthodes de travail et, dans certains cas, de déterminer le mode de diffusion de leurs résultats (CSE, 1995, p. 59).

C'est pour cette raison que la notion de liberté académique de Falconer (Landry, 2001) qui se limitait aux activités savantes au sein de l'université, fut élargie pour englober le grand spectre de la liberté d'expression, y compris le droit de critiquer l'université et ses dirigeants et le droit de parler ouvertement de questions controversées liées à l'ordre public, que ces questions tombent ou non dans le champ de compétence de la personne. En même temps, la gamme de comportements considérés acceptables de la part de quelqu'un qui jouit des privilèges associés à la liberté universitaire ne cessa de s’agrandir (Cameron, 1996a, p. 6).

\section{Et si la liberté académique était simplement piégée? Défis de l'orthodoxie}

Il s'agit ici de réfléchir à partir des éléments factuels pour étayer notre argumentation au sujet du caractère piégé de la liberté académique. Ainsi, dans les lignes qui suivent, nous reprenons quelques illustrations puisées dans les milieux universitaires congolais.

Comme le veut la tradition, après la proclamation des résultats de la première session d'examens, il est organisé un conseil de faculté. Celui-ci est généralement destiné à l'évaluation des activités passées et à la programmation des celles de l'année académique prochaine. C'est dans ce contexte qu'au cours d'une réunion du Conseil Facultaire (CONFAC) tenue le 12 août 2015 sous la présidence du Recteur de l’Université de Kisangani (UNIKIS), l'on a procédé à la nouvelle attribution des enseignements aux professeurs à la Faculté des sciences sociales, administratives et politiques (FSSAP). Cependant, avant d'y arriver, une évaluation a précédé cette attribution des cours sur demande du président de la séance. Il fallait évaluer globalement l'année académique passée (2014-2015) pour avoir une idée sur une éventuelle amélioration de la situation à l'année prochaine (2015-2016).

Concrètement, il est ressorti de cette évaluation, que la grande majorité des professeurs est consciente de la dégradation de l'éthique professionnelle et des conditions de travail à l'UNIKIS. Les professeurs ont épinglé plus de dix problèmes relevant du domaine de l'éthique, recoupant en grande partie les problèmes identifiés par eux-mêmes ou dans lesquels ils sont personnellement impliqués. Parmi ces problèmes, il y a lieu de citer la non-application de la loi dans certaines matières, le non-respect des horaires 
de cours par les professeurs ${ }^{26}$, le rapprochement exagéré entre professeurs et étudiants, la corruption, les abus dans la perception des droits d'auteur, la légèreté dans la cotation des étudiants, foulant ainsi au pied les exigences de la pédagogie universitaire qui constitue pourtant une condition sine qua none pour être nommé Professeur Associé (PA), le favoritisme, le manque de rigueur dans la conduite des travaux (les travaux de fin de cycle, les mémoires de licence ou de master, voire les thèses de doctorat). Face à toutes ces pratiques peu recommandables, nous pouvons bien nous poser la question de savoir quelle est la raison d'être d'une cellule d'assurance qualité dans les universités congolaises en général. Au regard des faits, il ressort nettement que cette cellule n'est qu'un nouveau site de dysfonctionnement institutionnel, car elle n'est pas en mesure de relever les défis dans ce secteur.

Examinant, par exemple, le premier problème épinglé par les enseignants de la Faculté de sciences sociales à l’Université de Kisangani, à savoir la non-application rigoureuse de lois et règlements dans certaines matières, il est intéressant de fournir ici quelques illustrations de défaillances dans la gestion académique et administrative du personnel. Dans le premier cas, un doctorant a été autorisé par le Comité de gestion (organe central de décision) de défendre sa thèse de doctorat en économie, sans pourtant avoir obtenu une distinction à la suite de son mémoire de spécialisation (D.E.S.=Diplôme d’études supérieures). Cela est une violation flagrante de la loi qui organise le doctorat dans les universités congolaises. Le deuxième cas concerne un professeur à qui un cours a été retiré par le Conseil de faculté en sciences sociales. Ce professeur, contre toute attente, s'est arrogé le droit de programmer le même cours, et l'a enseigné, défiant ainsi l'autorité décanale. Il n’en a jamais été reproché pour autant. Ces faits, manifestement gravissimes à l’Université, se déroulent sous la barbe des universitaires (professeurs), et personne n’en parle pour ne pas être identifié comme anarchiste. Par contre, à un autre enseignant qui n'a commis aucun abus, le Comité de gestion a infligé un blâme en guise de sanction malgré la carence des faits reprochés et sans lesquels la sanction perdrait sa légalité.

L’insubordination caractérisée vis-à-vis de l'autorité décanale, faute qui n’a pas été sanctionnée dans le premier cas, trouve ici un terrain d'application par pur sentiment. C'est une violation de la liberté académique car il s’agit d'une faute dépourvue d'existence factuelle et la sanction infligée frise de l'intimidation à l'égard du concerné qui devra adopter un profil bas pour éviter de s’attirer de nouveaux ennuis. Le Comité de gestion s'est contenté de faire plaisir à un ami professeur, Doyen de la Faculté, qui

${ }^{26}$ Comment peut-on expliquer qu'un cours de 45 heures puisse rester sur les horaires pendant deux mois voire trois, sans que le professeur titularisé n’en soit interpellé ? 
avait expressément demandé une sanction contre son collègue professeur, qui a toujours affirmé sa liberté académique. Il est pénible d'envisager l'hypothèse d'un oubli du Comité de gestion qui l'aurait entraîné à commettre une violation à la fois du règlement d'administration et de la liberté académique de l'enseignant incriminé. Peut-on parler d'une insubordination caractérisée sans l'existence d'un ordre ou d'une instruction donnée par un supérieur dont relève l'agent, ou sans que ce dernier pose un acte traduisant une révolte contre l'autorité supérieure ? Même en prenant l'hypothèse d'une loi que l'agent aurait violée, ceci ne correspondrait pas non plus à la qualification retenue : insubordination caractérisée vis-à-vis de l'autorité décanale.

C'est ici le lieu de souligner l'abus du pouvoir discrétionnaire qui laisse une trop grande marge de manœuvre à l'autorité d'apprécier la situation et de décider de façon unilatérale sur la qualification de tout fait, geste, écrit ou parole de l'agent visé pour fonder une sanction (Warren, 2011). La violation de la liberté académique est ici assurée faute de détermination expresse par la loi ou le règlement, des comportements spécifiquement identifiés comme fautes administratives. Toutes les incompréhensions suscitées par l'exercice des libertés académiques, dans un environnement où seul le silence des subalternes est visé par ceux qui détiennent une parcelle d'autorité, tendent ainsi à favoriser les violations desdites libertés et donc à créer un climat professionnel d'insécurité. André Mbata synthétise ces types de situations, généralement exploitées par les détenteurs du pouvoir, en ces termes :

«La responsabilité des universitaires dans la violation des libertés académiques se manifeste aussi dans leur intolérance contre des collègues qui ne partagent pas les mêmes thèses qu'eux. Des cas sont nombreux où, se moquant des libertés académiques, les professeurs imposent aux chefs de travaux, assistants et étudiants sous leur supervision leurs propres projets de recherche ou leur façon de penser. En raison de cette intolérance, certaines thèses et certains mémoires n'ont jamais été soutenus et d'autres travaux ont été très mal cotés parce que les professeurs ne s'y retrouvaient pas. Tout se passe comme si toute critique du collègue très bien placé et soutenu dans le corps ou du maître devrait condamner au bannissement intellectuel. De la part des enseignants, c'est aussi violer les libertés académiques que de ne pas vouloir se soumettre à la critique et d'apprécier les travaux des collègues et étudiants non pas sur la base de leurs mérites, mais sur des bases sentimentales comme l'appartenance à une même tribu, une même ethnie ou une même région. » (Mbata, 2005, p. 61-62).

Dans les universités congolaises en général, les chercheurs travaillent dans des conditions inhumaines (misère matérielle) qui, à leur tour, 
conduisent à l'effritement de valeurs morales (misère morale : corruption, par exemple) avant de déboucher inexorablement sur la misère intellectuelle, qui piège la liberté académique. Comment peut-on exercer la liberté académique dans un contexte où la vérité scientifique elle-même est étouffée, où le politique prime sur la science, où les critères même de sélection du personnel scientifique sont foulés au pied au bénéfice des avantages et repositionnements politiciens, où la misère sociale gangrène le secteur, et où il y a violation flagrante des franchises universitaires par les autorités politiques? Avec ces conditions inventoriées, les universités sont continuellement assaillies de sollicitations toujours renouvelées de la part de différents segments de la société, notamment du secteur politique, surtout durant ces dernières décennies.

Plusieurs demandes et recommandations provenant des autorités politiques en faveur notamment des députés (au niveau tant provincial que national) et autres acteurs politiques, favorisent leur nomination comme membres du personnel scientifique, sans moindre référence aux critères exigés par l’Université. En effet, Pour être nommé personnel scientifique dans les universités de la RDC, le candidat doit avoir fait une distinction au moins une fois pendant le cursus universitaire, et surtout en dernière licence. Il doit également être réputé de bonne moralité, et avoir un âge acceptable ... Sur ce dernier critère, par exemple, il est simplement regrettable de constater dans certaines circonstances que des candidats approchant la soixantaine, accèdent facilement au statut d'Assistant tout simplement parce qu'ils ont été recommandés par certains dignitaires du régime en place, quand des candidats, tout jeunes et remplissant les critères exigés, sollicitent en vain le même poste pour renforcer le personnel pourtant en nombre insuffisant au sein de nouveaux départements de la Faculté des sciences sociales de l’Université de Kisangani.

A la faveur du principe majoritaire prôné en démocratie, certains députés arrivés au parlement avec le niveau de diplômé de secondaire ont profité de cet environnement d'anarchie pour étudier dans les différentes institutions universitaires à travers le pays. Comme si cela ne suffisait pas, ces derniers ont poussé leur zèle jusqu'à devenir promoteurs (fondateurs) de leurs propres établissements d'enseignement supérieur pour mieux perpétuer leur hégémonie en signe de victoire dans l'espace universitaire.

Plusieurs acteurs politiques détenant le diplôme de licencié profitent du même contexte de désordres dans le secteur pour, non seulement accéder aux avantages liés au salaire accordé aux professeurs, mais également bénéficier de l'honneur dû aux professeurs. Nombre d'entre eux ont même développé des ambitions démesurées, en particulier celle de devenir enseignant à l’Université, sans le niveau requis pour y faire carrière. Dans ce contexte, désormais, "c’est le grade qui précède le savoir». Les 
enseignants-chercheurs potentiels seraient formés, non plus pour acquérir les connaissances nécessaires à la profession, mais juste pour avoir le diplôme. Il suffit, pour s'en convaincre, de jeter un regard critique, par exemple, sur l'organisation des séminaires de D.E.S. (Master). Les formateurs (professeurs qualifiés) se montrent de plus en plus préoccupés par les bénéfices économiques liés à cette prestation. Ils n’observent pas le volume horaire des cours sous prétexte que les candidats en formation sont présumés connaître le contenu desdits cours pour les avoir étudiés quand ils furent étudiants ou pour avoir été associés en tant que collaborateurs (Assistants ou Chefs de travaux) aux enseignements similaires destinés aux étudiants de premier ou de deuxième cycle. Pourtant, selon l'esprit du législateur congolais, les études de troisième cycle, notamment celles conduisant à l'obtention du titre de "Diplômé d'études supérieures », visent à former les spécialistes de l'enseignement supérieur et universitaire dans leurs filières d'études respectives. Qu'attendre donc d'une telle formation dans son format observé sur le terrain quand les étudiants terminent sans avoir approfondi ce à quoi le programme est destiné ? La systématisation de connaissances est ainsi renvoyée à l'autodidactie ex-situ ${ }^{27}$ dans un contexte où les bibliothèques font visage de parents pauvres, si pas simplement de pièces de musée.

La rigueur, ou mieux la «culture de l'excellence », qui sous-tendait la formation à l'Université n'est plus au rendez-vous ; on observe ainsi des arrangements autour de la recherche. Plusieurs mémoires de DES voire les thèses de doctorat sont produits, mais il faut reconnaître qu'un grand nombre de ces travaux sont réalisés en dehors de conditions régulières (Bongeli, 2015 ; Mawanzi, 2015 ; Amuri, Kasereka \& Kasereka, sous presse). Comme conséquence, il y a prolifération de mémoires de DES et de thèses de doctorat. L'évaluation de ces travaux peut, dans une large mesure, s'avérer fallacieuse parce que certains examinateurs n'évalueraient plus les travaux, mais se contenteraient de coter les individus en fonction de ce que ces derniers représentent à leurs yeux. André Mbata estime que tous ces faits sont illustratifs de violations de libertés académiques en RDC:

"Liée à la prostitution physique et intellectuelle qui a envahi les milieux universitaires et comme une forme de celle-ci, la corruption est également une pratique contraire à la promotion des libertés académiques. Celles-ci sont également violées lorsque l'inscription, l'obtention des diplômes, la nomination et la promotion dans le grade sont tout simplement monnayés. Par ailleurs, dans le contexte de la privatisation des établissements de l'ESU qui ne dit pas son nom, lorsque les étudiants doivent payer des primes aux enseignants, le fait

\footnotetext{
${ }^{27}$ En dehors du cadre ou site naturel dédié à la formation.
} 
pour certains enseignants de conditionner la réussite à une interrogation, un examen ou l'obtention d'une meilleure cote pour le travail de mémoire ou la dissertation de fin de cycle au payement par l'étudiant du syllabus élaboré par l'enseignant ou à la remise d'une prime illégale de correction constitue à mes yeux d'autres formes de violations de libertés académiques. » (Mbata, 2005, p. 63).

Comment peut-on parler de liberté académique quand la vérité scientifique devient une affaire des autres voire d'une autre époque ? Ces faits, pourtant scandaleux, ne peuvent être dénoncés par n’importe qui au nom de la liberté académique, craignant les réprimandes et les sanctions de tous ordres, tels l'enlèvement, l'intimidation, le châtiment corporel, ... et pourquoi pas la mort ? (Scholars at Risk, 2015). On peut donc dire que le contournement de l'orthodoxie éthique et académique dans les établissements d'enseignement supérieur et universitaire congolais est une des conséquences directes des pratiques illicites des universitaires eux-mêmes. Ici, l'exigence de conformité aux règles ne représente plus un enjeu capable de garantir la réimplémentation de l'éthique et la déontologie professionnelle parmi les chercheurs. Sur ces pratiques illicites dans l'arène académique, il est intéressant d'écouter encore André Mbata les décrire :

« Les libertés académiques sont violées lorsque l'admission à l'université ou dans un établissement d'enseignement supérieur, le passage des interrogations ou examens sont conditionnés par l'offre des services sexuels ou lorsque des «distinctions » sont distribuées, la nomination comme assistants ou chargés de cours, la promotion dans le grade et l'attribution d'autres avantages et même la reconnaissance ou l'exercice des droits se font en échange des services sexuels exigés des membres de la communauté universitaire de sexe féminin. » (Mbata, 2005, p. 63).

Pour illustrer avec vigueur la responsabilité de la communauté universitaire dans les violations des libertés académiques en Afrique en général, André Mbata reprend bon nombre de ces dénonciations poignantes de Claude Ake datant de 1994. En dépit de cette distance temporelle, les faits dénoncés sont toujours d'actualité, prenant parfois une tournure plus dramatique en RDC. Ainsi, peut-on lire:

«En tant que communauté universitaire, nous avons contribué de manière significative à la mort de la liberté académique en Afrique. (...) Lorsque l'étau des pressions a commencé à se resserrer contre nous, nous n'avons pas fait grand-chose pour défendre l'enseignement supérieur et la liberté académique. Certains d'entre nous ont coopéré de manière opportuniste avec les recteurs et autres représentants de l'État, pour mater les étudiants et d'autres collègues qui ont essayé de résister à l'assaut donné contre nos universités. Certains d'entre-nous qui ont 
rallié le gouvernement, sont devenus les défenseurs de l'attaque contre les universités et en ont profité pour se venger contre des collègues pour des motifs le plus souvent bien piètres... (...) L'assaut contre nous et notre liberté n'a pas donné lieu à un combat contre nos agresseurs mais plutôt contre nous-mêmes. (...) Nous sommes divisés par notre esprit partisan, nos rancunes et nos jalousies qui nous poussent à mettre en quarantaine ou à faire renvoyer les meilleurs d'entre-nous comme Achebe et Soyinka. » (Mbata, 2005, p. 60)

Par ailleurs, l'éveil de conscience des professeurs face à l'effritement de l'éthique et de la déontologie professionnelle ne se traduit pas par des pratiques innovantes capables de mettre fin à ce que nous pouvons appeler, sans exagérer, un suicide collectif ou une servitude volontaire. Leur prise de conscience répond à une logique qui leur est propre et qui reflète à la fois leurs intérêts et positions en tant qu'acteurs sociaux, guidés par des motivations diverses. Ainsi, les universitaires attribuent-ils la responsabilité du relâchement des exigences éthiques et académiques à une gouvernance défaillante générale (malgouvernance de l'Etat), ce qui les empêche, eux aussi, d'agir efficacement de manière à renverser les vapeurs dans leur secteur d'activité. L'opinion dominante est celle qui répugne à admettre l'absolutisme éthique d'hier que soutenait Albert Einstein, un célèbre physicien du $20^{\mathrm{e}}$ siècle : "Relativity applies to physics, not ethics " (« La relativité s'applique à la physique, pas à l'éthique ») (Holzer \& Schwester, 2011, p. 359).

La relativité improvisée en RDC dans le domaine sensible de l'éthique, qui cautionne en fait l'impunité des comportements inacceptables dans l'univers académique, a de fâcheux précédents associés avec le régime Mobutu. En effet, il a été rapporté par Library of Congress Country Studies et CIA World Factbook (US), que l'euphémisme adopté dans le langage de l'incurie sous Mobutu a consacré l'institutionnalisation du vol et de la corruption ; ceci s'est passé dans un contexte où l'action énergique et nécessaire de l'Etat en vue du redressement des torts était pourtant attendue depuis longtemps. C’est ce qui transparaît dans cette critique du discours du président Mobutu prononcé en 1977:

"The widely used Lingala phrase, yiba moke, meaning "steal just a little," did not originate in popular conversation but in the text of a speech by President Mobutu in 1977. He was speaking out against runaway corruption and advised his countrymen that they should "steal just a little" or, perhaps, "steal wisely" and that they should invest the proceeds inside the country rather than stowing it abroad." (Library of Congress Country Studies \& CIA World Factbook, 1993). 
Comme on peut s’en rendre compte, le fait pour le président Mobutu d'enjoindre à ses compatriotes de « voler juste un peu » (yiba moke) ${ }^{28}$, qui ne correspond guère à l'interdiction de voler, est bien symptomatique d'un ordre social en déclin. Le vol, un acte moralement et légalement incorrect, donc répréhensible, ne saurait bénéficier de tolérance et ce, quelle que soit la quantité de biens emportés par son auteur (Ilunga, 1999 ; Svara, 2015). L’on ne saurait non plus considérer comme acceptable ou un moindre mal, le fait que des biens volés soient réinvestis au pays plutôt qu’à l’étranger. Progressivement, il s’est développé un vocabulaire dépénalisant et légitimant le vol : «Au Zaïre, on ne vole pas, on déplace » ; « il n’y a pas de voleurs, mais uniquement des “déplaceurs.” » Ce dernier terme, qui n’existe pas dans la langue française, est une invention dans le langage populaire congolais de la dérision, soulignant que l'acte de voler sans exporter les biens était toléré et dépouillé de tout caractère pénal. Des biens volés dans la capitale et emmenés en province ou d'une province vers une autre, ou simplement du bureau ou de l'entreprise publique pour la résidence ou la ferme d'un agent public, étaient cyniquement considérés comme simples changements d'adresse physique destinés à faciliter les services aux autres citoyens, membres de la même communauté nationale habitant un même pays.

Le problème tient cependant au fait que ces faits, pourtant anciens et ancrés, déjà décriés par des universitaires courageux-constituent une réalité avec laquelle il semble que nous soyons appelés à vivre désormais ils s'installent et même s'imposent dans un état de vide normatif, à la fois juridique, éthique et déontologique (Rocher, 1999). Dans cette posture problématique, il est évident que l'excellence dans les universités congolaises se révèle de plus en plus comme un attribut discutable. A juste titre, V. Cleemput Oswald (2012) pense que la baisse du niveau de l'enseignement supérieur et universitaire de la RDC est un fait connu. Les principales contraintes en sont la prolifération et l'émiettement des universités et des instituts supérieurs, les problèmes aigus d'infrastructures, d'équipements et de matériels didactiques, les problèmes du personnel enseignant, les difficultés de relève académique. Les causes de cette situation, ajoute-t-il, sont connues et sont à situer au niveau de la mauvaise gestion académique, administrative et financière.

L’on découvre également un bilan globalement sévère de l'enseignement supérieur congolais fait à partir de données empiriques couvrant l'Est et l'Ouest du pays. Ledit bilan est résumé en ces termes :

Le rapport d'identification (2009) du VLIR-UOS souligne clairement les problèmes du secteur de l'Enseignement Supérieur et Universitaire

\footnotetext{
${ }^{28}$ Yiba moke vient du lingala, l'une des quatre langues nationales du pays.
} 
: l'absence de l'autorité de l'Etat, le faible financement du secteur, le nombre d'établissements, les infrastructures, équipements didactiques et ressources humaines. En ce qui concerne les ressources humaines, le rapport souligne le vieillissement et la sous-qualification généralisée du personnel académique et scientifique. La situation est confirmée en 2013 : le secteur « est caractérisé par un désengagement quasi-total de l'Etat sur le plan budgétaire, et par l'inadéquation entre le financement et les objectifs de l'Enseignement, de la Recherche et des Services de qualité à la communauté» (Grega, Mayira \& Dhaene, 2013, p. 23).

Evoquant la problématique de la mondialisation et les enjeux de réforme de l'enseignement supérieur, W. Experton (2012) constate que les besoins de qualification et la demande pour l'enseignement supérieur sont en forte croissance en Afrique. Toutefois, l'Afrique ne peut retirer les bénéfices de cet investissement en capital humain que si les individus sont bien formés et employés. Aeneas Chapinga Chuma, un haut fonctionnaire onusien de l'Organisation Internationale du Travail (OIT) le souligne si fort en parlant du paradoxe de la croissance économique en Afrique :

« À l'heure actuelle, le grand écart persiste entre les compétences attendues sur le continent et la nature des formations administrées, laissant sur le carreau des bataillons de jeunes qui ne sont pas employables. Le dialogue entre les patrons d'industrie et le secteur éducatif doit être plus étroit. Aussi, il faut que les pays africains se lient davantage les uns aux autres, et que l'intégration économique régionale accélère. La libre-circulation ne doit pas s'arrêter aux biens, mais doit être étendue aux personnes et aux compétences. C'est une lacune importante. » (Polle, 2017)

Comme annoncé plus haut, prétendre imposer l'orthodoxie, à la fois sur le plan éthique et sur le plan académique dans l'espace universitaire congolais en mutation, présuppose de se poser la question (et d'y répondre) sur la gouvernance générale de la société congolaise dont l'université ne représente qu'une des principales fenêtres d'observation.

Plusieurs problèmes cependant peuvent être associés à une mauvaise gouvernance dans le secteur de l'enseignement supérieur en RD Congo. Les plus critiques se résument comme suit: (i) la prévalence de la gestion informelle, (ii) l'emprise de la politique sur le secteur de l'enseignement supérieur (sa politisation), (iii) le foisonnement des règlements et les difficultés d'application, (iv) l'incapacité à agir sur les problèmes, (v) la corruption, (vi) le népotisme, (vi) le manque de clarté dans la prise de décision, (vii) des lignes d'autorité et responsabilités confuses. Globalement, tous ces problèmes n'attendent que d'être résolus par des acteurs ayant un sens de responsabilité et des réflexes éthiques très développés. Les problèmes soulèvent la nécessité de repenser le leadership dans le secteur de 
l'enseignement supérieur en mettant un accent particulier sur les établissements publics. En raison de leur nombre, de leur profonde identification avec l'intérêt général et surtout de l’ampleur de leur fragilité actuelle, ces organisations méritent une grande attention de tous les acteurs concernés. Ces derniers sont identifiés par leur qualité de parties prenantes (stakeholders) dans la production des politiques reflétant un leadership effectif et transformateur (Van Wart, 2015 ; Howlett, Ramesh, \& Perl, 2011).

\section{Pour conclure : Que faire ?}

Il est fondamental de rappeler, de prime abord, qu'aucune campagne de sensibilisation ne saurait provoquer la rupture des pratiques peu recommandables dominant le secteur de l'enseignement supérieur congolais si des réformes courageuses voire impopulaires mais salutaires, ne sont pas entreprises en amont.

Il est aussi vrai que, dans le contexte actuel de la RDC, engager une réflexion critique sur une question aussi sensible que l'orthodoxie éthique et académique, sonne comme un excès de zèle, un acte d'ingratitude à l'égard de la Mère nourricière (Alma Mater) voire une trahison du culte de nationalisme défensif que célèbrent encore des milliers de compatriotes. Il faut néanmoins relever le fait que le glacis protecteur que représente ce nationalisme fanatique, n'est qu'une preuve de la naïveté que bon nombre d'universitaires congolais perpétuent, même au péril de l'institution avantgardiste et séculaire appelée Université.

Pour que la liberté académique devienne une réalité dans l'espace universitaire congolais, certaines conditions devraient être mises en place et accompagner les pratiques innovantes susceptibles de juguler les pratiques marginales qui gangrènent le secteur. Il faudrait considérer, à titre principal, le renforcement des mesures d'application du code d'éthique professionnelle à l'égard des universitaires congolais. Ceci est fondamental dans la mesure où il est inacceptable de justifier la prévalence des maux par la seule absence de meilleures conditions matérielles de vie et de travail pour les enseignantschercheurs. Théophile Mbemba Fundu di Luyindu a conscience de l'impératif d'améliorer ces conditions de vie et de travail des enseignantschercheurs. Aussi rapporte-t-on que l'enseignant en général, et l'enseignant d'université en particulier sur ses conditions de travail, n'a pas été oublié dans la réforme en cours. Car, il est à la fois la porte d'entrée et de sortie pour l'amélioration de la qualité de l'enseignement national. Et la moyenne d’âge des professeurs dépassant les 60 ans, le gouvernement va reformuler la politique d'administration des bourses d'études de manière à promouvoir l'excellence et la relève académique (Forum des As, 2015).

Il y a lieu de penser également à la promotion de l'autonomie des universités (différencier la science de la politique), de leur gestion 
académique, budgétaire et des ressources humaines, à la redéfinition et à la revalorisation de la carrière universitaire, à la dépolitisation du secteur de l'enseignement supérieur, et bien sûr, à la mise en place d'une gouvernance démocratique. Réfléchissant sur l'arrimage de la RD Congo au processus $\mathrm{LMD}^{29}$, Alphonse Maindo (2012) fait remarquer que la RDC est un pays où l'on croit que légiférer suffit à résoudre des problèmes. Ce dont la patrie de Lumumba a besoin, ajoute-t-il, c'est l'application des textes organisant la vie collective, quelles que soient leurs qualités et leur pertinence ${ }^{30}$.

Dans la même perspective, il faudrait reconnaître que, même si les enseignants-chercheurs (universitaires) doivent être interpellés sur leurs devoirs (responsabilité sociale), leurs conditions matérielles de vie et de travail ne sont pas de nature à favoriser l'application scrupuleuse des principes éthiques et professionnels pour un exercice effectif de la liberté académique en tant que droit. La misère matérielle induirait la misère morale et la misère intellectuelle, piégeant ainsi la liberté académique. Fort de cette évidence, il devient urgent de penser aussi à l'amélioration des conditions de travail des universitaires congolais, pour enclencher avec optimisme le processus d'une mise en œuvre orthodoxe de la liberté académique au sein de leurs institutions.

En outre, il faudrait éviter que se réédite le scénario rapporté par Marie-Soleil Frère à propos d'un séminaire sur l'éthique tenu à Accra (Ghana) en septembre 1999. Frère signale, en effet, la réaction d'un journaliste ghanéen qui suggère de relativiser le caractère orthodoxe de l'éthique, mettant en exergue le contexte particulier de nécessité (survie): «I can practise ethics of course, but I can't eat ethics ! ( « Je peux respecter la déontologie bien sûr, mais je ne peux pas manger la déontologie ! ») (Frère, 2000, p. 266). Explicitement, l'auteur avance une explication qui corrobore l'argumentaire de la majorité des citoyens, même parmi les plus instruits, confrontés à la crise endémique en Afrique :

« Cette dérive est encouragée, dans la plupart des pays africains, par l'instauration d'un modèle social de plus en plus inégalitaire où les revenus des privilégiés, accédant à la manne de l’aide étrangère, à la rente d'Etat ou aux bénéfices des récentes privatisations recommandées par les institutions financières internationales, sont sans

${ }^{29}$ LMD qui signifie Licence-Maîtrise-Doctorat est un programme découlant du Processus de Bologne entrepris en Europe et qui, depuis quelques années, est suivi par la plupart des pays africains de l'espace francophone dont la RDC. Il s'inscrit, en fait, dans la logique des réformes initiées sur le continent dans le domaine de l'enseignement supérieur.

${ }^{30}$ En effet il existe plusieurs textes (lois, codes, ...) rédigés en très bon français, mais le problème réel qui se pose c'est leur application. C'est justement à ce niveau que les institutions concernées doivent parvenir à prendre des mesures courageuses qui peuvent être impopulaires, mais qui pourront se révéler salutaires. Par exemple, parvenir à châtier les cas de flagrance à l'université (corruption, bagarres entre enseignants, haine ethnique...). 
commune mesure avec ceux de l'énorme majorité des citoyens. » (Frère, 2000, p. 266-267).

De même, sous le Zaïre de Mobutu, des propos fatalistes de la part des étudiants ont été rapportés concernant leur perception de l'incurie ambiante constatée dans la gestion des affaires publiques. Il est révélé le fait que les comportements déviants (détournement de deniers publics ou d’équipements destinés à l'usage public) sont tolérés, même dans les couches supérieures de la société, insinuant l'attitude paradoxale des élites-les étudiants, futurs dirigeants ou leaders d'opinion-qui s'accommodent curieusement de l'état anomique qu'inspire la gouvernance atypique de leur pays :

"This sense extends to the upper reaches of society; university students in Kisangani would at times exonerate those responsible for thefts of public funds with the phrase s'il y a l'anarchie, profitez-en, (if there's anarchy, profit from it)” (Library of Congress Country Studies \& CIA World Factbook, 1993).

Finalement, il faut l'avouer, c'est ce genre de situations, bien documentées par les instances internationales et mises à la portée du monde entier via Internet, qui ont fait, font et feront encore pour longtemps le lit des malheurs congolais. Il s’agit de comprendre que la réputation est une ressource stratégique essentielle à la formation du soft power d'un Etat ou d'une communauté nationale. A l'ère digitale, plus rien ne restera longtemps caché. Et les Congolais en général, instruits ou non, sont le plus souvent traités à l'étranger, en Afrique ou ailleurs, en fonction de l'image écornée que leur pays d'origine, la RDC, renvoie au monde entier. Il est urgent de repenser les manières d’être et de faire pour espérer reconquérir le crédit de traiter avec les autres en tant que partenaires, en commençant par le secteur stratégique par essence, celui de l'enseignement supérieur. Il semble évident que l'absence d'un enseignement spécialisé en leadership ${ }^{31}$ dans les organisations du secteur public, est une lacune fondamentale qui, en fin de compte, serait identifiée comme la cause majeure des échecs enregistrés au cours des décennies postindépendance en RDC.

L'orthodoxie éthique et académique n'est pas un mythe, même si elle demeure encore une terre lointaine ; c'est plutôt le refus du changement qui représente une option dangereuse pour une nation en pleine crise. Le mal congolais, au-delà de la crise politique actuelle, passe pour non seulement profond, mais surtout singulier sur le continent africain voire au monde (Butcher, 2008). Comment sortir de cette grande et longue nuit? Seule assurance, c'est que la longueur de la nuit (l'état d'anomie) ne constitue guère une fatalité contre le dynamisme des sociétés humaines. La liberté

${ }^{31}$ Celui-ci ne se confond pas avec le management des organisations. 
académique, avec ses soubassements éthiques impliquant des responsabilités sociales pour tout enseignant ou chercheur, devrait constituer aujourd'hui une garantie qui permette au génie intellectuel, culturel et spirituel de l'élite congolaise d’éclore. Par conséquent, cette liberté d’esprit devrait inspirer la société congolaise et ses dirigeants politiques en vue du rétablissement rapide du consensus social, actuellement rompu.

\section{Remerciements}

Nous exprimons notre reconnaissance aux organisations Scholar Rescue Fund-Institute of International Education (SRF-IIE) et Scholars At Risk (SAR) pour leur soutien universel à la défense, la protection et la promotion des libertés académiques dans le monde, et à Global University Project—DR Congo pour son appui en documentation et logistique.

\section{References:}

1. Amuri Misako, F., Kasereka Mahalibo, J. \& Kasereka Kaputu, A. (sous presse). La recherche en sciences sociales à l'Université de Kisangani (1983-2016) —RD Congo. Répertoires des mémoires de DES, thèses de doctorat et publications de I'IRSA, collection Géopolitique mondiale, Paris, L’Harmattan.

2. Arendt, H. (1991). Juger. Sur la philosophie politique de Kant, Paris, Seuil.

3. Bergeron, J. (2012). «L’Université de la RDC: Une identité nationale dans l'espace mondial de la connaissance ? ", dans Maindo Monga Ngonga, A. et Kapagama Ikando, P (dir.). L'Université en chantier en RD Congo: Regards croisés sur la réforme de l'enseignement supérieur et universitaire, Paris, Karthala, (pp. 7285).

4. Bongeli Yeikelo Ya'Ato, E. (2015). Education en République démocratique du Congo. Fabrique de cerveaux inutiles? Paris, L'Harmattan.

5. Butcher, T. (2008). Blood River: The Terrifying Journey through the World's Most Dangerous Country, New York: Grove Press.

6. Cameron, D.M. (1996a). « Academic Freedom and the Canadian University ", Paper commissioned by the Association of Universities and Colleges of Canada, Research Files, Vol. 1, No 3, (pp.1-11).

7. Cameron, D.M. (1996b). « La liberté universitaire et l'université canadienne », Dossier de recherche, vol.1, no 3, pp. 1-11. (Version française de Cameron, 1996a).

8. Cleemput Oswald, V. (2012). «Promotion de la qualité dans les universités congolaises et compétitivité sur le plan international », dans Maindo Monga Ngonga, A. et Kapagama Ikando, P. (dir.) 
L'Université en chantier en $R D$ Congo: Regards croisés sur la réforme de l'enseignement supérieur et universitaire, Paris, Karthala, (pp. 56-71).

9. Durkheim, E. (1967). De la division du travail social (livres I, II, III), $8 \mathrm{e}$ éd., collection : Bibliothèque de philosophie contemporaine, Paris, PUF.

10. Durkheim, E. (1897). Le suicide. Etude de sociologie (Livres I, II, III), Paris, PUF.

11. Experton, W. (2012). "Quel mode de gouvernance pour l'enseignement supérieur en République Démocratique du Congo ? », in Maindo Monga Ngonga, A. et Kapagama Ikando, P. (dir.) L'Université en chantier en $R D$ Congo: Regards croisés sur la réforme de l'enseignement supérieur et universitaire, Paris, Karthala, (pp. 85-98).

12. Forum des As. (2015). “'Ouverture de l'année académique 2015-2016 en RDC. Le ministre Théophile Mbemba indexe l'UNIKIN pour ses années élastiques,'”16 octobre. http://7sur7.cd/new/ouverture-delannee-academique-2015-2016-en-rdc-le-ministre-theophilembemba-indexe-lunikin-pour-ses-annees-elastiques.

13. Frère, M.-S. (2000). "Les mots et le pouvoir : le nouveau vocabulaire de la presse privée dans les régimes de transition en Afrique ", Hermès, no 28, (pp. 257-270).

14. Grega, P. Mayira, A. \& Dhaene, C. (2013). Evaluation intérimaire de la Coopération Universitaire Institutionnelle en RD Congo, VLIRUOS, juillet.

15. Holzer, M. \&Schwester, R. W. (2011). Public Administration: An Introduction, New York and London: M. E. Sharpe.

16. Howlett, M., Ramesh, M. \& Perl, A. (2011). Studying Public Policy: Policy Cycles \& Policy Subsystems, 3rd Edition, New York: Oxford University Press.

17. Ilunga Kabongo. (1999). "Le marécage ou le Zaïre des années 1990”, Canadian Journal of African Studies/Revue canadienne des Etudes africaines, Vol. 33, No. 2/3, Special Issue: French-Speaking Central Africa: Political Dynamics of Identities and Representations, (pp. 410-431).

18. Journals Consortium. (2015). African Universities and Higher Institutions Ranking.

19. Landry, S. (2001). « La liberté académique et l'autonomie universitaire : un recueil de citations ", Les Cahiers de la FQPPU, ${ }^{\circ}$ 6, (pp. 22-66).

20. Leroux, G et Vidricaire, A. (1996). Vingt-cinq ans de syndicalisme universitaire: éléments d'histoire et enjeux actuels, Montréal, SPUQ., 
(pp. 9-11).

21. Library of Congress Country Studies \& CIA World Factbook. (1993). "Democratic Republic of the Congo: Theft and Bribery" http://www.photius.com/countries/congo_democratic_republic_of_th e/society/congo_democratic_republic_of_the_society_theft_and_brib ery.html. Accessed 05-04-2017.

22. Maindo Monga Ngonga, A. (2012). «Et si le LMD passait préalablement par la réforme de l’homme ? », dans Maindo Monga Ngonga, A. et Kapagama, Ikando, P (dir.). L'Université en chantier en $R D$ Congo : Regards croisés sur la réforme de l'enseignement supérieur et universitaire, Paris, Karthala.

23. Maindo Monga Ngonga, A. et Kapagama Ikando, P (dir.). (2012). L'Université en chantier en RD Congo: Regards croisés sur la réforme de l'enseignement supérieur et universitaire, Paris, Karthala.

24. Marie-Françoise et Fave-Bonnet. (2002). «Conflits de missions et conflits de valeurs: la profession universitaire sous tension ", Connexions : logiques conflictuelles des modèles universitaires, no 78, février, (pp. 11-30).

25. Mawanzi Manzenza, Th. (2015). L’Université de Kinshasa en quête de repères, Paris, L’Harmattan.

26. Mbata Betukumesu Mangu, A. (2005). «Libertés académiques et responsabilité sociale des universitaires en République démocratique du Congo ", Journal of Higher Education in Africa / Revue de l'enseignement supérieur en Afrique, Vol. 3, No. 2, Special Issue: Academic Freedom: Global Challenges, African Experiences, pp. 3581. Stable URL: http://www.jstor.org/stable/24486249 Accessed: 0504-2017 05:52 UTC

27. Otemikongo Mandefu, J. et Losumbe Bondondo, J. (2001). « Politique d'essaimage des établissements d'enseignement supérieur et universitaire dans la ville de Kisangani », Revue de l'IRSA, nos 78, novembre, (pp. 65-79).

28. Polle, B. (2017). «Aeneas Chapinga Chuma, directeur Afrique de l’OIT : "La croissance africaine ne fournit pas assez de travail”" ", Jeune Afrique du 05 avril. http://www.jeuneafrique.com/424925/economie/aeneas-chapingachuma-directeur-afrique-de-loit-croissance-africaine-ne-fournitassez-de-travail/, consulté le 05 avril 2017.

29. Rocher, G. (1999). « Entre un passé qui s’estompe et un avenir aux formes incertaines », allocution d'ouverture du Colloque La recherche universitaire et les partenariats), Université, vol. 8, no 2.

30. Savage, D.C. (2001). « Liberté académique et autonomie universitaire dans le cadre de la commercialisation de la recherche », 
conférence prononcée lors du colloque Mutation du financement universitaire et commercialisation de la recherche, organisé par le Comité de la liberté académique et de l'autonomie universitaire (CLAAU) de la FQPPU, tenu le 2 mars 2001.

31. Scholars at Risk. (2015). Free to Think: Report of the Scholars at Risk Academic Freedom Monitoring Project.

32. Svara, J. (2015). The Ethics Primer for Public Administrators in Government and Nonprofit Organizations, Burlington: Jones \& Bardett Learning.

33. Unesco. (1998). Conférence mondiale sur l'enseignement supérieur, Paris.

34. Van Wart, M. (2015). Leadership in Public Organizations: An Introduction, $2^{\text {nd }}$ Edition, London and New York: Routledge.

35. Warren, K. F. (2011). Administrative Law in the Political System, 5th Edition, Boulder: Westview Press. 